\title{
Did the Universe Have a Chance?
}

\author{
C. D. $\mathrm{McCoy}^{*}$
}

14 December 2018

\begin{abstract}
In a world awash in statistical patterns, should we conclude that the universe's evolution or genesis is somehow subject to chance? I draw attention to alternatives that must be acknowledged if we are to have an adequate assessment of what chance the universe might have had.
\end{abstract}

\section{Cosmology and Chance}

Although cosmology, the study of the universe's evolution, has largely become a settled province of empirically-grounded physics, the more distant shores of cosmogony, the study of the origin of the universe, continue to fascinate the speculative imaginations of physicists and philosophers alike. Of course, many people believe that science has settled questions about the origin of the universe too, at least on a popular understanding of the well-known and well-confirmed big bang model of the universe, for, as the popular imagination has it, the universe itself began with an event, the eponymous "big bang." Putting it this way is not quite right however. As the general theory of relativity has it, there was no such first event; rather, what the big bang universe has is a "past singularity," and a singularity is a global property of spacetime, not some actual event in spacetime. That the big bang universe is singular does, however, entail that any observer's temporal past is finite-but, nevertheless, despite having a finite age, it does not have a first moment (just as there is no first positive real number).

At very early times the big bang universe evolved through a sequence of extremely hot, dense states (decreasing in temperature and density with increasing time). Cosmologists often

*Acknowledgments: I am grateful to Heather Demarest for commenting on this paper at the 2018 Society for the Metaphysics of Science Conference in Milan. Thanks also to Richard Dawid and Alex Franklin for discussion, and to the audience at the 2018 Biennial Meeting of the Philosophy of Science Association in Seattle.

$\dagger$ Department of Philosophy, Stockholm University, Stockholm, Sweden. Email: mailto:casey.mccoy@philosophy.su.secasey.mccoy@philosophy.su.se 
understand this sequence of initial hot, dense states as the apt referent of the term "big bang." On this characterization, the big bang is not thought of as the beginning but instead as a very early stage of the universe's evolution, in which case the big bang falls properly within cosmology, not cosmogony, since it concerns the evolution of the universe. One reason to follow the cosmologists' interpretation is that, while our physical evidence indicates that we may be highly confident that the big bang account is correct back to a very early time, it becomes progressively less compelling as we go farther and farther back in time. At three minutes it is nigh incontrovertible; but earlier, say one second, the story becomes fairly dubious, due to uncertainty about the physics involved. In using "big bang" in this way, cosmologists therefore avoid committing themselves to a particular cosmogonical story while still being able to maintain that the big bang's cosmological account is largely correct.

Of course, those so inclined may also thereby find time enough to encourage speculations about the origin of the universe. ${ }^{1}$ Indeed, philosophers and physicists have seized the opportunity afforded by this window of uncertainty to make substantive explanatory posits, especially with an eye towards explaining the statistical patterns we observe in the world. Perhaps most conspicuous is the supposition that the universe began in a low entropy state (Penrose, 1989; Albert, 2000; Wald, 2006; Carroll, 2010; Feynman, 2017). According to statistical mechanics, this means assigning an appropriate probability measure to the time associated with this state. Now, since this measure must be objective in order for it to do the explanatory and descriptive work of statistical mechanics, it evidently needs to be grounded in objective features of the world. While there are various proposals for substantiating the objectivity of physical probabilities, the most metaphysically-inclined will take the straightforward road of grounding probability assignments of this state ontically (Loewer, 2004; Demarest, 2016), which is to say, to take them to be chances (Briggs, 2010).

Should we say that the universe had a chance then? Drawing on my previous work (McCoy, 2018a, b, forthcoming a, b, c), I suggest that the answer is not at all clear yet. Many authors promote their views in the context of limited alternatives, but the possibilities are far larger than it might seem from the literature. I focus here on two contrast classes to the question that must be recognized: first, concerning determinism and indeterminism, and, second, concerning different implementations of chances in an indeterministic theory.

\footnotetext{
${ }^{1}$ Were there previous stages to the universe, before the big bang? What actually brought the universe into existence? What was the character of the initial happening (should it in fact truly exist)? See (Callender and McCoy, forthcoming) for a survey of some current thinking on questions like these, related to time and the origin of the universe.
} 


\section{Some Metaphysical Preliminaries}

By the world metaphysicians generally mean something like "the maximally inclusive entity whose parts are all the things that exist." Terminology does vary, of course. This particular rendering comes from Schaffer $(2010,33)$, who prefers to call this entity the cosmos. As it happens, cosmologists do not usually call their object of study the cosmos; more commonly they say that they study the universe. They seldom have much to say about how the universe which they study relates to the more "worldly" concerns of the metaphysician. There are occasional exceptions however. Harrison provides a notable one with his text Cosmology: The Science of the Universe, in which he discusses the philosophical and historical dimensions of cosmology (and cosmogony) alongside the more standard material of physical cosmology. What metaphysicians call the world Harrison designates the Universe. The cosmology of scientific cosmologists, according to Harrison, is the study of universes: scientific models of the Universe (Harrison, 2000, Ch. 1), which are used to describe especially its large-scale structure and the evolution thereof.

This is a practically useful distinction to make, one which I will adopt in the following. By the world I intend, following the practice of metaphysicians, the locus of (principally) metaphysical questions (concerning what Harrison calls the Universe): Is the world deterministic? Is it chancy? These are the kinds of questions primarily at issue in this paper. By the universe I designate, following the practice of cosmologists, the locus of principally physical questions concerning what Harrison calls the Universe: How did the Universe begin? How will it end? These latter are questions to which a cosmological model, like the big bang model, should suggest or indicate some answer.

I do not mean, of course, to introduce any sharp distinction between science and metaphysics by differentiating universe and world. Indeed, when one asks whether the world has some feature, naturalistic-minded metaphysicians of science look first to scientific models of the Universe to help decide the question. For instance, when addressing the question whether the world is deterministic, Wüthrich claims that "this metaphysical question deflates into the question of whether our best physical theories entail that the world is deterministic or indeterministic" (Wüthrich, 2011, 366).

Naturalistic discussions of determinism generally adopt the approach favored by Wüthrich, and so will I. Let determinism stand for the thesis that the world is deterministic. Then, following (Lewis, 1983, 360), a world is deterministic just when the laws of that world are deterministic. To determine whether the laws of the universe are deterministic, we must look to our theories of which those laws are part and ask whether those laws (taken together) should be considered deterministic. It is by no means a straightforward matter to decide whether a given physical theory is deterministic. Even the classic example of deterministic physics, Newtonian mechanics, appears to admit of many indeterministic counterexamples to its purported determinism (Earman, 1986; Norton, 2008). General relativity too permits indeterministic 
behavior in the form of causal pathologies (closed timelike curves) and, if the hole argument is to be believed, is hopelessly rife with indeterminism (Earman and Norton, 1987).

If one excludes outlandish counterexamples and interpretations, however, then one might reasonably regard both theories as essentially deterministic, at least for all practical purposes. As standard cosmological models, such as the big bang universe, are models of the general theory of relativity (our best theory of the universe, at least at large scales), it would then follow that our universe is deterministic, in which case it seems that physical cosmology tells us unequivocally that the universe never had a chance (McCoy, 2018a). Following the exhortation of naturalistic metaphysicians to answer metaphysical questions by looking to our best physical theories, we may then come to the conclusion that the world too is not at all chancy.

Certainly, general relativity is not our best theory all physical phenomena considered. Indeed, our best theories in many contexts are probabilistic theories, like quantum mechanics and statistical mechanics. These theories, one might expect, are indeterministic through and through, because of the probabilistic element. Various interpreters of these probabilistic theories seek to evade any seemingly inherent indeterminism, however, by characterizing probabilities as merely epistemic or subjective (which approaches I will be mostly setting aside here for reasons of space) or else by attempting to portray them as fully deterministic theories (as in the Bohmian interpretation of quantum mechanics) (Sklar, 1993; von Plato, 1994; Guttmann, 1999; Bricmont et al., 2001; Beisbart and Hartmann, 2011; Ben-Menahem and Hemmo, 2012).

Philosophers have raised serious concerns, though, over how one can truly understand probabilities in deterministic theories that seem to depend on them for their empirical content, an issue that has been termed the "paradox of deterministic probabilities" (Loewer, 2001; Winsberg, 2008; Lyon, 2011) in statistical mechanics. The objectivity of these probabilities must be derived from some objective ground. One possibility, the one with which I am principally concerned in this paper, is that they are directly grounded in some element of randomness in the nature of things, that is, they are genuine chances. Lewis insisted that deterministic chances, at least, are incoherent: "to the question of how chance can be reconciled with determinism, or to the question of how disparate chances can be reconciled with one another, my answer is: it can't be done" (Lewis, 1986, 118). He was surely right about this. The basic problem is that the world cannot both be chancy and deterministic, for the ontic character of these probabilities simply entails indeterminism, the contrary of determinism (McCoy, forthcoming c, §1).

Several authors have nevertheless offered accounts of "deterministic chance" in order to resolve the aforementioned paradox (Glynn, 2010; Eagle, 2011; Lyon, 2011; Briggs, 2015; Emery, 2015). These either run afoul of the problem just mentioned or are not really accounts of deterministic chance (but rather characterize some kind of merely epistemic probability) (McCoy, forthcoming c, §2). As far as I can see, there is only one successful reconciliation of chance and determinism, namely that defended by Loewer (2001) in the context of statistical 
mechanics. It is seldom recognized by interpreters, however, that there is no reconciliation in the sense of a simultaneous compatibility of chance and determinism in Loewer's account - that is not possible, just as Lewis says.

Instead, Loewer's account shows us how deterministic laws can co-exist with indeterministic laws within a theory. The source of all probabilities in statistical mechanics, according to Loewer, is in an initial chance distribution over microscopic states of affairs. After the initial time these states of affairs evolve deterministically. Crucially, although for almost all times evolution is deterministic in Loewer's account, it is not so for all times. There is, in effect, an initial chance event, which is where the indeterminism of the theory appears. Recall that a deterministic theory is a theory whose laws are deterministic, not a theory whose laws are mostly deterministic or operate deterministically for almost all times. Thus, Loewer's account does not attempt to confute the metaphysical conflict of chance and determinism (McCoy, forthcoming c, §3). Rather, it finds a way to harmonize both aspects in a single world.

Loewer is a Humean, though, and does not believe that the chances and laws of probabilistic theories exist independently of categorical events or facts (Loewer, 1996). According to the Humean (of Loewer's stripe), they are consequences of the best systematizations of the occurrent facts, as maintained in Lewis's "best systems account" of laws and chances (and variations thereof) (Loewer, 2004). Humean chances are thus ontologically reducible. Demarest offers a non-reductionist tweak to Loewer's account by invoking a "robustly metaphysical account of chance" (Demarest, 2016, 256) in the face of Loewer's reductionism. She claims that such chances are compatible with determinism, and indeed they are when compatibility is rendered as the mere co-existence of indeterministic and deterministic laws in a single theory (which cannot, of course, operate at the same time).

Despite their differences, they both regard the objective probabilities of physics as ontic probabilities - in other words, as chances, not as merely epistemic probabilities (Winsberg, 2008; McCoy, forthcoming c). Thus, Loewer's and Demarest's accounts, of initial chances in otherwise deterministic universes, have the consequence that worlds to which these accounts apply had a chance. ${ }^{2}$

\section{Did the Universe Really Have a Chance?}

Loewer's and Demarest's apparently consistent interpretations of statistical physics can be read to suggest that the universe had a single, initial chance. Is it so? To clarify the standing of any such claim about chanciness in the world, I will draw attention in this section to two important and salient contrast classes which can be associated with the general question, "did the universe

\footnotetext{
${ }^{2}$ Did our world have a chance? Although statistical mechanics is not a candidate for a fundamental theory of the universe, quantum mechanics is, so if one of their accounts is right, then, happily, we could say that our world had a chance after all.
} 
have a chance?" In the metaphysical preliminaries of the previous section, these contexts were already implicitly introduced. The first is whether the cosmological theory is deterministic or indeterministic. ${ }^{3}$ The second is whether the probabilities of an indeterministic cosmological theory are probabilities of initial conditions or of the dynamics. ${ }^{4}$

\subsection{Determinism and Indeterminism}

A primary motivation for Loewer and Demarest is to give a philosophical undergirding for statistical physics explanations of statistical patterns in the world. When we ask whether the world had a chance, however, our concern goes beyond the ontological possibilities of statistical physics, even granting our naturalistic method, to all (physically) viable ontological possibilities. For this reason we should wonder whether statistical patterns in empirical data by themselves provide good evidence for indeterministic (specifically, probabilistic) theories rather than deterministic theories. If a deterministic theory can ground these patterns, then it may be nothing other than a pragmatic preference that leads us to favor (ostensibly indeterministic) statistical physics explanations.

Certain statistical patterns do indeed appear to favor a deterministic explanation and some an indeterministic one. Consider the following example: the pattern of bombings in London on the first day of the Blitz during World War 2. ${ }^{5}$ The clustering of the bombings around certain locations strongly suggests a primarily causal explanation, namely that the Luftwaffe pilots were aiming at specific targets and their bombs roughly landed near where they aimed. Consider next a related example: the pattern of V2 rocket strikes around London late in the war. ${ }^{6}$ Here the pattern looks distinctively random, and indeed V2 rockets were notoriously unreliable in a way that suggests a dominant element of randomness involved in the associated explanation of the pattern. Of course, looks can be deceiving: there may well be a perfectly deterministic underlying description of this pattern when one inquires further into the details—say, into the particular environmental conditions and rocket parts. ${ }^{7}$

Even in the context of physical theories, where we can be more precise about the issue of determinism, it is far from clear that statistical patterns tell us unequivocally how they should be described (and explained) theoretically. As Werndl (2009) shows, building on the claims of (Suppes, 1993), there is a demonstrable observational equivalence of indeterministic theories and deterministic theories for the same set of observations (i.e., statistical patterns). Specifically, she shows that for any stochastic system there is an observationally equivalent deterministic system and vice versa. That is, there is an underdetermination by empirical

\footnotetext{
${ }^{3}$ See, for example,(Suppes, 1993; Werndl, 2009, 2013; Wüthrich, 2011; Hoefer, 2016).

${ }^{4}$ See, for example, (Maudlin, 2007, 2011; Wüthrich, 2011).

${ }^{5}$ See http://bombsight.org/\#12/51.5049/-0.0896

${ }^{6}$ See https://www.wrsonline.co.uk/big-ben-rocket-strikes/1944-v2-rocket-attacks-map/

${ }^{7}$ See (Pynchon, 1973) for an alternative deterministic account of the V2 rocket strike pattern.
} 
evidence at the level of theory, which undermines a conclusion for or against chancy, indeterministic behavior in the world on the basis of empirical evidence.

Such theoretical underdetermination by empirical evidence will not be terribly surprising to anyone acquainted with the philosophy of science literature. So is there perhaps other grounds to which we may appeal? For example, if we widen our scope of evidence to include explanatory considerations, do we then have reason to favor deterministic or indeterministic theories? Werndl (2013) claims that we do. She argues that indirect evidence can break the underdetermination, since a theory that gives a unified description of a collection of models is preferable to one that does not. Although she does not portray her argument as an inference to the best explanation, it is a natural interpretation, for what makes a unifying theory preferable is, intuitively, that it gives a better explanation than one that does not. Of course, we can continue the debate whether unification is a legitimate epistemic justification or is merely a pragmatic one. That is too large a debate to venture into here. But it does appear that there is a significant burden on the unificationist to defend this as adequate grounds, for, to put the point briefly, there must be a strong connection between unification and truth for it to count as epistemically justifying in general, yet it is difficult to see what this connection could be (much as it is difficult to see what connection there could be between simplicity and truth).

Admittedly, this is only one way in which explanatory considerations might be thought to bear on the determinism question. And while I am pessimistic that any universal scheme can succeed, I do believe that explanatory considerations are epistemically justifying in certain contexts (as I argue in (McCoy, forthcoming b)). If that is right, then we can and do have adequate epistemic grounds to favor particular theories. Nevertheless, to be fully successful, any such argument would have to rule out, or make relatively implausible, any alternative. And unfortunately, philosophers who attempt to draw a metaphysical conclusion on the basis of some particular theory have done little to demonstrate that this underdetermination is sufficiently limited to secure it.

\subsection{Implementations of Chance}

A second contrast to consider concerns how chances are implemented in a probabilistic physical theory, namely as initial chances or dynamical chances. This is a context class that is emphasized by, among others, (Maudlin, 2011; Wüthrich, 2011), taking as a model the idea that dynamical theories explain via two things: laws of evolution and initial conditions (Hempel, 1965). So, on the one hand, probabilities may all have their source in initial chances, as in the accounts of Loewer and Demarest described above, while having an otherwise deterministic dynamics; on the other hand, probabilities may by dynamical, in the sense that the laws of evolution are probabilistic laws, specifying only probabilities for state transitions. As intuitive as such a characterization may be, however, it is misleading in at least a couple of ways.

This becomes clear when one realizes that there are many ways to implement chances in a 
physical theory. All one must do, as (Loewer, 2001) shows us by example, is neatly separate when the indeterministic laws are operative and when the deterministic laws (if any) are operative. Loewer chooses to locate all the indeterminism in one particular place - the initial time - but one could equally locate it at another time, at many times, at all times, or even at no time at all (outside of time, as it were).

Now, the initial time may seem particularly privileged as a time for locating a single chance event. But why? In the case where the world is otherwise deterministic, locating the chance event at any time whatsoever will determine the world's history completely. So, as far as determination goes, there is nothing special about initial chances. Something else, then, is required in order to favor the initial time. The most intuitive is to say that there is an arrow of time in accord with which the laws are causal, productive, or governing. Maybe in this case only the initial time is an acceptable time to locate a chance event. However, even granting that, it should be clear that there even being an initial time is a rather special case. If the universe were temporally finite to the past but lacked an initial time, as in the big bang universe, then locating the chance event at the "initial time" is not possible. Locating it at any time near to the "beginning" would be just as arbitrary as locating it at any time whatsoever. Similarly, if the universe is past infinite, then locating the chance event at any time whatsoever is completely arbitrary.

To avoid this kind of unpalatable arbitrariness, one might suggest locating the chance event "outside of time." That is, rather than locating a chance event at some definite time, one takes the chanciness of the world to concern the realization of a particular, complete deterministic history from a collection of more or less probable possible histories. In opting for this interpretation one might even truly say that the universe never had a chance, since the "chance event" is not "in" the universe. Then again, one should probably not attach too much to there being no literal chances in the universe in this case, for the realization of a particular deterministic history is also precisely what the initial chance account achieves (as the deterministic evolution of the system guarantees a bijection between initial conditions and complete histories).

In any case, any of these "single chance" interpretations invites the oft-invoked (in cosmology) picture of the (blind and unskilled) Creator throwing a dart (Wald, 2006, 396) or pointing a pin (Penrose, 1989, 442) at the set of possible universes, thereby picking out the initial conditions of the universe. The message behind drawing such pictures is often that such an interpretation is purely metaphysical (in a pejorative sense). Whether this is cause for concern or not, drawing together all of a theory's indeterminism into a single random trial is not the only option. As said, chancy behavior could be inherent in the dynamics instead; that is, the theory's dynamics is stochastic. There are various examples of implementing chances in this way in the foundations of quantum mechanics (Ghirardi, 2016): indeterministic behavior can happen, for example, at discrete times, as in the GRW interpretation, or continuously, as in the CSL interpretation. Other examples arise in classical statistical mechanics, especially 
non-equilibrium statistical mechanics (Wallace, 2014; Luczak, 2016; McCoy, 2018b).

There is, however, a third way to implement chances in a physical theory: implementing the chancy behavior in the observable properties of a system, as I have recently suggested (McCoy, forthcoming a). In the aforementioned "stochastic dynamics" approach the indeterminism of the theory is an indeterminism of states - state transitions are indeterministic - whereas the observable properties of a system are fully determined by the state. The alternative "stochastic observables" approach, by contrast, maintains a deterministic state dynamics and instead implements indeterminism in the observable outcomes-which observable outcome is realized is a matter of chance.

One might categorize the stochastic observables interpretation as a species of dynamical chance, saying, for example, that there is no physical difference between indeterministic state evolution and indeterministic realization of observables. Perhaps that is right. But if that is right, then with at least equal reason one ought to count "initial" chances as dynamical chances, since they involve an indeterministic dynamical transition at some time (for example, an initial time). Do we recognize a single category of chance then? Two? Three? At the very least, it is not so clear that the division of chances into initial conditions and dynamical laws is so apt. At the very least, there are manifold possibilities that should be recognized for implementing chances in a physical theory.

\section{Conclusion}

Although I began with a discussion of some aspects of cosmology which motivate the idea that the universe had a single chance, almost all discussion of physical cosmology has long since dropped out of consideration. Indeed, by now it should be clear that contemporary physical cosmology is little relevant to this idea and issue. Although the conceptual opaqueness of probability is a match for the opaqueness of the beginning of our universe, this is surely not a sufficient reason to locate chances there. Whatever genuine reasons interpreters of statistical physics actually have, I have argued that it is important to recognize a variety of coherent ways to implement chances in a probabilistic physical theory-it is not just a choice between initial chances and stochastic dynamics. Moreover, although in all of these chance implementations we may say that the universe (so interpreted) is chancy, we must also acknowledge alternatives at higher levels of interpretation. In particular, we must acknowledge that there may be unconceived deterministic alternatives to our indeterministic probabilistic physical theories - at least until these can be clearly ruled out.

\section{References}

Albert, David. 2000. Time and Chance. Cambridge: Harvard University Press. 
Beisbart, Claus, and Stephan Hartmann, eds. 2011. Probabilities in Physics. Oxford: Oxford University Press.

Ben-Menahem, Yemima, and Meir Hemmo, eds. 2012. Probability in Physics. Berlin: Springer.

Bricmont, Jean, Detlef Dürr, Maria Galavotti, Giancarlo Ghirardi, Francesco Petruccione, and Nino Zanghì, eds. 2001. Chance in Physics. Berlin: Springer.

Briggs, Rachael. 2010. “The Metaphysics of Chance.” Philosophy Compass 5:938-952.

Briggs, Rachael. 2015. "Why Lewisians Should Love Deterministic Chance." In A Companion to David Lewis, eds. Barry Loewer and Jonathan Schaffer, 278-294. New York: John Wiley \& Sons.

Callender, Craig, and Casey McCoy. Forthcoming. "Time in Cosmology." In The Routledge Handbook for Philosophy of Physics, eds. Alistair Wilson and Eleanor Knox. London: Routledge.

Carroll, Sean. 2010. From Eternity to Here. New York: Plume.

Demarest, Heather. 2016. "The Universe Had One Chance.” Philosophy of Science 83:248-264.

Eagle, Antony. 2011. “Deterministic Chance.” Noûs 45:269-299.

Earman, John. 1986. A Primer on Determinism. Dordrecht: D. Reidel Publishing Company.

Earman, John, and John Norton. 1987. "What Price Spacetime Substantivalism? The Hole Story." The British Journal for the Philosophy of Science 38:515-525.

Emery, Nina. 2015. "Chance, Possibility, and Explanation.” The British Journal for the Philosophy of Science 66:95-120.

Feynman, Richard. 2017. The Character of Physical Law. Cambridge: The MIT Press.

Ghirardi, Giancarlo. 2016. "Collapse Theories." In The Stanford Encyclopedia of Philosophy, (Spring 2016 ed.), ed. Edward Zalta. URL:

¡https://plato.stanford.edu/archives/fall2018/entries/qm-collapse/ $i$ (accessed 2018-12-14).

Glynn, Luke. 2010. "Deterministic Chance." The British Journal for the Philosophy of Science 61:51-80. 
Guttmann, Yair. 1999. The Concept of Probability in Statistical Physics. Cambridge: Cambridge University Press.

Harrison, Edward. 2000. Cosmology: The Science of the Universe (2nd ed.). Cambridge: Cambridge University Press.

Hempel, Carl. 1965. Aspects of Scientific Explanation. New York: The Free Press.

Hoefer, Carl. 2016. "Causal Determinism.” In The Stanford Encyclopedia of Philosophy (Spring 2016 ed.), ed. Edward Zalta. URL: ¡https://plato.stanford.edu/archives/spr2016/entries/determinism-causal/i (accessed 2018-12-14).

Lewis, David. 1983. "New Work for a Theory of Universals." Australasian Journal of Philosophy 61:343-377.

Lewis, David. 1986. Philosophical Papers, Volume 2. Oxford: Oxford University Press.

Loewer, Barry. 1996. "Humean Supervenience.” Philosophical Topics 24:101-126.

Loewer, Barry. 2001. "Determinism and Chance." Studies in History and Philosophy of Modern Physics 32:609-620.

Loewer, Barry. 2004. "David Lewis's Humean Theory of Objective Chance." Philosophy of Science 71:1115-1125.

Luczak, Joshua. 2016. "On How to Approach the Approach to Equilibrium.” Philosophy of Science 83:393-411.

Lyon, Aidan. 2011. "Deterministic Probability: Neither Chance nor Credence." Synthese 182:413-432.

Maudlin, Tim. 2007. "What Could be Objective About Probabilities?" Studies in History and Philosophy of Modern Physics 38:275-291.

Maudlin, Tim. 2011. "Three Roads to Objective Probability." In Probabilities in Physics, eds. Claus Beisbart, and Stephan Hartmann, 293-319. Oxford: Oxford University Press.

McCoy, Casey. (2018a). "The Implementation, Interpretation, and Justification of Likelihoods in Cosmology." Studies in History and Philosophy of Modern Physics 62:19-35.

McCoy, Casey. 2018b. "Interpretive Analogies Between Quantum and Statistical Mechanics.” [Preprint] URL: ¡http://philsci-archive.pitt.edu/id/eprint/14479i (accessed 2018-12-14). 
McCoy, Casey. Forthcoming a. "An Alternative Interpretation of Statistical Mechanics." Erkenntnis.

McCoy, Casey. Forthcoming b. "Epistemic Justification and Methodological Luck in Inflationary Cosmology." The British Journal for the Philosophy of Science.

McCoy, Casey. Forthcoming c. "Stability in Cosmology, from Einstein to Inflation." In Thinking About Spacetime, eds. Beisbart, Claus, Tilman Sauer, and Christian Wüthrich. Basel: Birkhäuser.

Norton, John. 2008. "The Dome: An Unexpectedly Simple Failure of Determinism." Philosophy of Science 75:786-798.

Penrose, Roger. 1989. The Emperor's New Mind. Oxford: Oxford University Press.

Pynchon, Thomas. 1973. Gravity’s Rainbow. New York: Viking Press.

Schaffer, Jonathan. 2010. "Monism: The Priority of the Whole." The Philosophical Review 119:31-76.

Sklar, Lawrence. 1993. Physics and Chance. Cambridge: Cambridge University Press.

Suppes, Patrick. 1993. "The Transcendental Character of Determinism." Midwest Studies in Philosophy XVIII:242-257.

von Plato, Jan. 1994. Creating Modern Probability. Cambridge: Cambridge University Press.

Wald, Robert. 2006. "The Arrow of Time and the Initial Conditions of the Universe." Studies in History and Philosophy of Modern Physics 37:394-398.

Wallace, David. 2014. "Probability in Physics: Stochastic, Statistical, Quantum." In Chance and Temporal Asymmetry, ed. Alistair Wilson, 194-220. Oxford: Oxford University Press.

Werndl, Charlotte. 2009. "Are Deterministic Descriptions and Indeterministic Descriptions Observationally Equivalent?" Studies in History and Philosophy of Modern Physics 40:232-242.

Werndl, Charlotte. 2013. "On Choosing Between Deterministic and Indeterministic Models: Underdetermination and Indirect Evidence.” Synthese 190:2243-2265.

Winsberg, Eric. 2008. "Laws and Chances in Statistical Mechanics." Studies in History and Philosophy of Modern Physics 39:872-888.

Wüthrich, Christian. 2011. "Can the World Be Shown to Be Indeterministic After All?" In Probabilities in Physics, eds. Claus Beisbart, and Stephan Hartmann, 365-389. Oxford: Oxford University Press. 